\title{
Electrically driven single photon source based on a site-controlled quantum dot with self-aligned current injection
}

Cite as: Appl. Phys. Lett. 101, 211119 (2012); https://doi.org/10.1063/1.4767525

Submitted: 22 October 2012 • Accepted: 31 October 2012 • Published Online: 20 November 2012

W. Unrau, D. Quandt, J.-H. Schulze, et al.

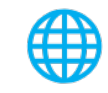

\section{ARTICLES YOU MAY BE INTERESTED IN}

Electrically driven quantum dot-micropillar single photon source with $34 \%$ overall efficiency Applied Physics Letters 96, 011107 (2010); https://doi.org/10.1063/1.3284514

Invited Review Article: Single-photon sources and detectors

Review of Scientific Instruments 82, 071101 (2011); https://doi.org/10.1063/1.3610677

Electrically driven quantum dot single-photon source at $2 \mathrm{GHz}$ excitation repetition rate with ultra-low emission time jitter

Applied Physics Letters 102, 011126 (2013); https://doi.org/10.1063/1.4774392

田QBLOX

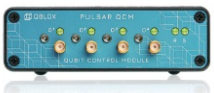

1 qubit
Shorten Setup Time Auto-Calibration More Qubits

\section{Fully-integrated} Quantum Control Stacks Ultrastable DC to $18.5 \mathrm{GHz}$ Synchronized $<<1$ ns Ultralow noise

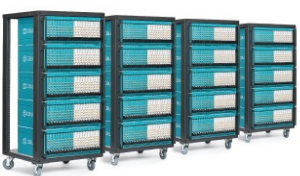

100s qubits

visit our website > 


\title{
Electrically driven single photon source based on a site-controlled quantum dot with self-aligned current injection
}

\author{
W. Unrau, D. Quandt, J.-H. Schulze, T. Heindel, T. D. Germann, O. Hitzemann, \\ A. Strittmatter, S. Reitzenstein, U. W. Pohl, and D. Bimberg \\ Institut für Festkörperphysik, Technische Universität Berlin, Hardenbergstrasse 36, D-10623 Berlin, Germany
}

(Received 22 October 2012; accepted 31 October 2012; published online 20 November 2012)

\begin{abstract}
Electrical operation of single photon emitting devices employing site-controlled quantum dot (QD) growth is demonstrated. An oxide aperture acting as a buried stressor structure is forcing sitecontrolled QD growth, leading to both QD self-alignment with respect to the current path in vertical injection pin-diodes and narrow, jitter-free emission lines. Emissions from a neutral exciton, a neutral bi-exciton, and a charged exciton are unambiguously identified. Polarization-dependent measurements yield an exciton fine-structure splitting of $(84 \pm 2) \mu \mathrm{eV}$ at photon energies of $1.28-1.29 \mathrm{eV}$. Singlephoton emission is proven by Hanbury Brown and Twiss experiments yielding an anti-bunching value of $\mathrm{g}^{(2)}(0)=0.05$ under direct current injection. (C) 2012 American Institute of Physics.

[http://dx.doi.org/10.1063/1.4767525]
\end{abstract}

Future quantum-communication systems depend on the availability of low-cost sources of single polarized photons or entangled photon pairs. Low-dimensional semiconductor materials such as quantum dots are suitable candidates for electrically driven single-photon sources (SPS) since they show very narrow emission linewidth and can be easily integrated with mainstream semiconductor diode technology. ${ }^{1-3}$ Pure single-photon characteristics as expressed by second order auto-correlation values $\mathrm{g}^{(2)}(0)<0.02$ have been demonstrated. ${ }^{4}$ Electrical operation of QD-based single photon emitters has been previously demonstrated with $\mathrm{g}^{(2)}(0)<0.02$ and repetition rates up to $1 \mathrm{GHz} .^{5-7}$ Moreover, the exploitation of quantum electrodynamics such as the Purcell effect in QDmicrocavities allows one to strongly enhance the extraction efficiency of SPSs and overall efficiencies of up to $34 \%$ have been reported for electrically pumped devices., ${ }^{8,9}$ Quantum optical systems strongly benefit from QDs with their low excitonic dephasing rates ${ }^{10}$ and narrow emission linewidths. Excitonic transitions exhibiting linewidths down to few $\mu \mathrm{eV}$ at low temperatures are obtained, if QDs are embedded into defect-free matrix material such that no jitter or spectral diffusion induced by stochastic fluctuations of electric fields in the QD environment occurs. Thus, QD-based single photon emitters are key elements of future quantum communication systems and are already used for quantum key distribution. ${ }^{3,11,12}$

One important problem of QD-based SPS prohibiting broad use is related to the random spatial distribution of QDs on the growth surface resulting from the probabilistic nature of the self-organized Stranski-Krastanow growth mode, which is typically used during epitaxy. The SPS device yield is consequently low, and the performance of QD-SPSs remains arbitrary as long as QD alignment is not precisely controlled. In recent years, much effort has therefore been directed towards so-called site-controlled growth of QDs. ${ }^{13-18}$ The common task is to locally modify the free energy of the growth surface in order to increase the probability for QD nucleation at predefined sites. Many techniques for site-controlled growth have been developed which provide the intended deterministic nucleation characteristics. A few groups also demonstrated electrical operation of diode structures with site-controlled QDs embedded into the intrinsic region of pin-diodes. ${ }^{19-21}$ Best reported values show excitonic linewidths of $170 \mu \mathrm{eV}$ and photon auto-correlation values with $\mathrm{g}^{(2)}(0)=0.4 .^{21}$

The surface patterning technique used to achieve sitecontrolled growth has a significant impact on the optical quality of the QDs. Depending on the way of patterning and the subsequent growth procedure, defects may be created in close vicinity to optically active QDs. Such defects typically contribute to severe line broadening due to spectral diffusion. $^{22}$ As one possible solution to the problem of patterninginduced defects we recently proposed and demonstrated sitecontrol of QDs using a buried stressor layer. This technology employs the strain field of a buried oxide aperture to trigger the nucleation of QDs within the aperture. By embedding QDs into this defect-free environment, their optical properties have become now comparable to the ultimately high optical quality of QDs randomly grown on planar surfaces. Additionally, this approach is very attractive for the processing of deterministic devices since perfect self-alignment of the site-controlled QDs to the vertical current path as confined by the oxide aperture is obtained. In this work, we demonstrate electroluminescence (EL) spectra of single QDs integrated into a pin-diode structure via buried stressor, featuring linewidths of excitons down to $25 \mu \mathrm{eV}$ and electrically driven single-photon emission showing anti-bunching with $\mathrm{g}^{2}(0)=0.05$.

A two-step metal organic vapor phase epitaxy procedure is applied to embed site-controlled QDs into a vertical pindiode structure as shown in Fig. 1. During the first step, the bottom part comprising a 20-period n-doped AlGaAs/GaAs distributed Bragg-reflector (DBR) and the stressor structure are grown. For the stressor, a $120 \mathrm{~nm}$ thick AlGaAs/AlAs/ AlGaAs sandwich structure buried by GaAs is deposited. Afterwards, arrays of circular mesas with diameters ranging between $22.0 \mu \mathrm{m}$ and $24.8 \mu \mathrm{m}$ in $200 \mathrm{~nm}$ steps are realized by standard photolithography and dry etching. An etching depth of $300 \mathrm{~nm}$ is chosen to yield access to the AlAs layer via the mesa sidewalls. Subsequently oxide apertures are 
(a)

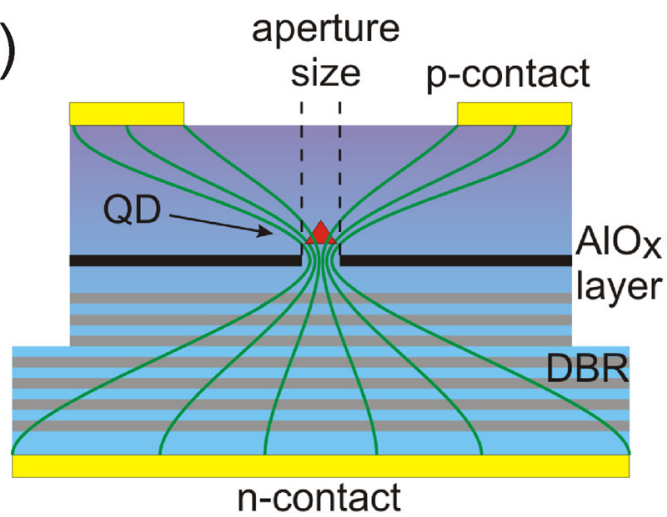

(c)

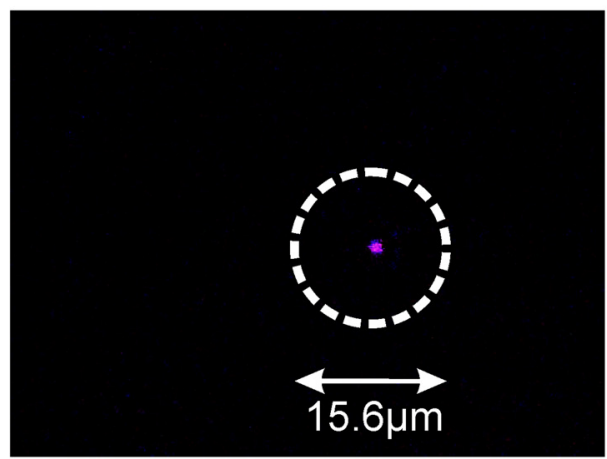

(b)
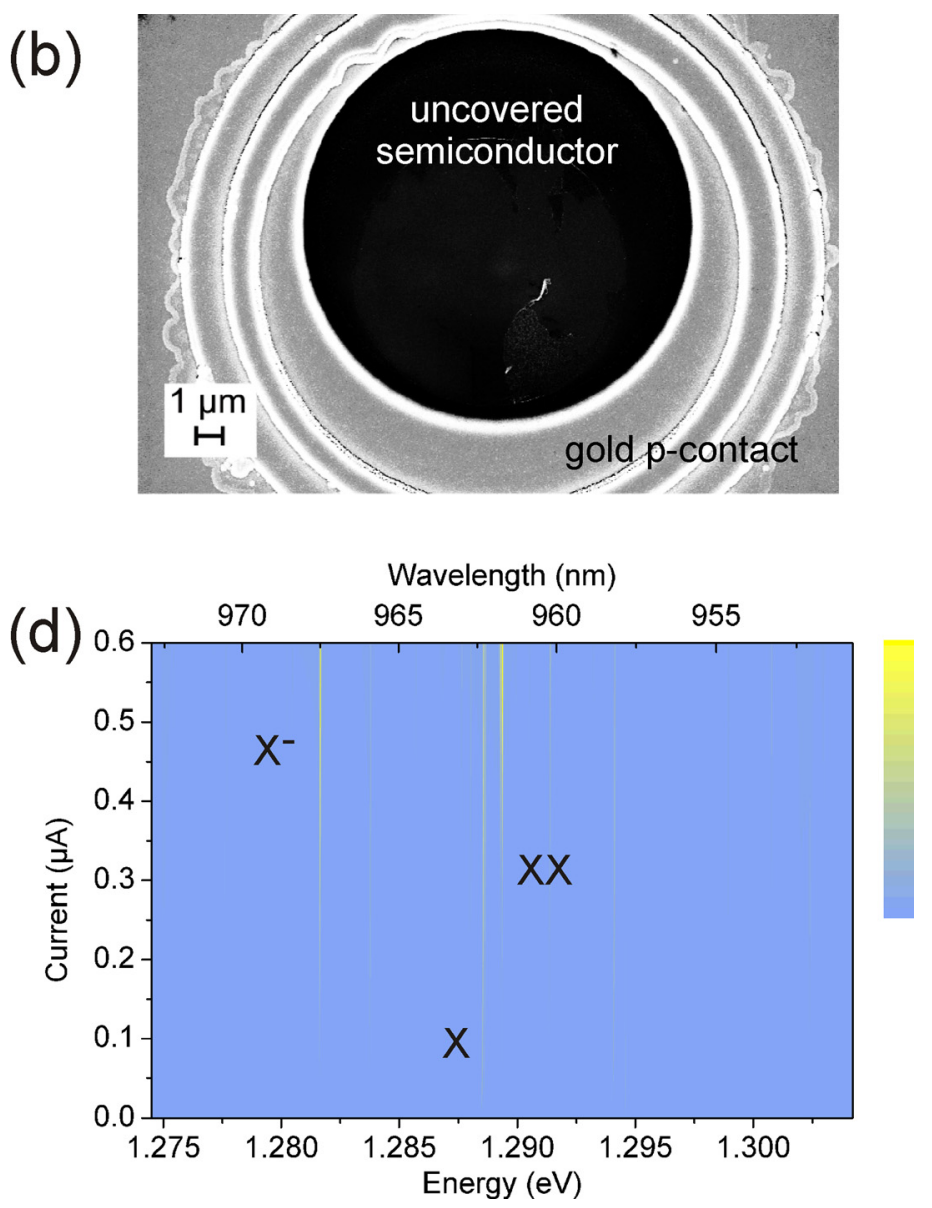

FIG. 1. (a) Schematic cross section of a device indicating QD position, aperture size, and current flow (green lines); (b) SEM image of the top side of the device area. In view: uncovered semiconductor, p-contact metallization, and several circular topographic profiles from first mesa etch, mesa diameter reduction, and $\mathrm{SiN}_{\mathrm{x}}$ passivation opening; (c) EL emission as recorded by a digital camera using an IR filter; (d) electroluminescence spectra recorded from the aperture region as a function of the injection current. Emission from excitons (X), bi-excitons $(\mathrm{XX})$, and charged excitons $\left(\mathrm{X}^{-}\right)$are labeled.

formed using selective oxidation of the AlAs layer in a furnace under water steam/nitrogen atmosphere at $\mathrm{T}=420^{\circ} \mathrm{C}$. In situ control of the oxidation rate is used to stop the process when sub- $\mu \mathrm{m}$ apertures are obtained. Arrays of mesa structures are found to exhibit open apertures within the oxide layer for $\mathrm{d}_{\text {mesa }}>23 \mu \mathrm{m}$. A detailed description of this method has recently been reported. ${ }^{23}$

The second epitaxial growth sequence is initiated by oxide desorption at $720^{\circ} \mathrm{C}$ using arsine stabilization. Prior to the QD layer, about $50 \mathrm{~nm}$ of GaAs is deposited at $685^{\circ} \mathrm{C}$. Parameters for QD growth are independently calibrated on planar and reference mesa templates. The InGaAs QD layer and a GaAs cap layer are grown at $485^{\circ} \mathrm{C}$. Except for the layer thickness which is close to the $2 \mathrm{D} / 3 \mathrm{D}$ transition of the Stranski-Krastanow growth regime, all other parameters are equal to those applied for QD layers optimized for laser devices. ${ }^{24,25}$ The planar QD density is tuned to below $1 \times 10^{8} \mathrm{~cm}^{-2}$ to provide good selectivity between QD nucleation on the aperture region and surrounding areas. After GaAs cap layer deposition, the growth temperature is raised to $600^{\circ} \mathrm{C}$. Then, another $50 \mathrm{~nm}$ thick undoped GaAs layer and a $110 \mathrm{~nm}$ p-doped GaAs contact layer are grown.

Important for site-controlled nucleation of QDs is the tensile surface strain within the aperture region, which reduces the lattice mismatch between InGaAs/GaAs. Tensile strain is generated upon volume reduction of the oxidized layer. This lattice distortion of $1 \%-2 \%$ lowers locally the free energy for QD growth, thus preferring QD nucleation above the oxide apertures. ${ }^{23}$ By choosing an appropriate layer thickness and aperture size, precise control of the QD number within the aperture region is possible.

For device fabrication, each mesa diameter is reduced by $5 \mu \mathrm{m}$ via dry etching, which removes any parasitic deposits on mesa side-walls and also QDs located at the mesa edge where an area of parasitic QD nucleation is present. The structure further comprises a backside n-contact metallization. Front-side insulation using $\mathrm{SiN}_{\mathrm{x}}$ is applied prior to topside p-contact metallization with circular openings of around $15 \mu \mathrm{m}$ aligned to the mesa center. Fig. 1(a) illustrates schematically the cross section of the device structure. As displayed, the aperture within the oxide layer defines simultaneously the QD position and the vertical current path through the device. Thereby, optimum charge carrier injection into a target QD is achieved. Fig. 1(b) depicts a top view of a fully processed device taken by a scanning electron microscope (SEM).

Optical characterization of the device was performed in a high-resolution micro-electroluminescence ( $\mu$-EL) setup at low temperatures $(10-50 \mathrm{~K})$. Bonding of devices onto chip carriers is required prior to mounting into a continuous-flow helium cryostat. The emission is collected through a $20 \times$ microscope objective with a numerical aperture of 0.4 providing a focal 
spot size of about $3 \mu \mathrm{m}$. The $\mu$-EL emission is dispersed by a spectrometer with a focal length of $0.75 \mathrm{~m}$ and a spectral resolution of $25 \mu \mathrm{eV}$. Coarse and fine positioning of devices is achieved by a combination of stepper motors and piezoelectric actuators. Photon auto-correlation measurements are performed using a fiber coupled Hanbury-Brown and Twiss (HBT) configuration based on a 50:50 multimode fiber beam splitter and two Si-avalanche photo diodes with $0.8 \mathrm{~ns}$ timing resolution.

Fig. 1(c) shows low-temperature EL of a device at a current of $1 \mu \mathrm{A}$ recorded by a digital camera attached to the $\mu$-EL setup. The emission is filtered by a $930 \mathrm{~nm}$ edge-type optical filter to suppress contributions at higher energies. The total output area of the device is indicated by a dashed white circle. As can be seen, long-wavelength $(>930 \mathrm{~nm})$ emission is generated from a region above the current confining aperture (in the following it is referred to as aperture region) which is also the area of site-controlled QD growth. Diode-like current-voltage characteristics with an onset voltage of $\mathrm{V}_{\mathrm{ON}}=1.4 \mathrm{~V}$ and a reverse saturation current $<0.1 \mathrm{nA}$ are found (not shown). From overview spectra taken between 1.270 and $1.320 \mathrm{eV}$ the onset of QD emission at injection currents of $0.01 \mu \mathrm{A}$ is determined to be located in a spectral window between $1.280 \mathrm{eV}$ and $1.295 \mathrm{eV}$. Fig. 1(d) shows successive $\mu$-EL spectra in the spectral range of 1.275$1.305 \mathrm{eV}$ taken from the aperture region for increasing currents from $0.01 \mu \mathrm{A}$ to $0.60 \mu \mathrm{A}$ at a temperature of $12.5 \mathrm{~K}$. For the device under test with a mesa diameter of $24.6 \mu \mathrm{m}$ an aperture size of $0.2-0.4 \mu \mathrm{m}$ is estimated from the oxidation rate and time.

In Fig. 2(a), individual $\mu$-EL spectra at different injection currents are shown in a waterfall presentation for better display of the power dependence of individual lines. At an injection current of $0.01 \mu \mathrm{A}$, only one excitonic transition is visible whereas up to nine optical transitions are observed at higher injection currents $(0.60 \mu \mathrm{A})$. The low total number of emission lines is attributed to a very low QD number directly above the aperture region. All lines exhibit narrow spectral linewidths below $50 \mu \mathrm{eV}$, with best values of $25 \mu \mathrm{eV}$ (still resolution limited), which constitutes a major progress as compared to previous reports on site-controlled QD growth. ${ }^{13-17,20,21}$ The high spectral purity of the emission lines also facilitated the identification of several characteristic electronic transitions associated with the same QD, namely, the bi-exciton (XX), the neutral exciton $(\mathrm{X})$, and the negatively charged exciton $\left(\mathrm{X}^{-}\right){ }^{26}$ Moreover, fine structure splitting (FSS) of the exciton level system is well resolved. At $0.01 \mu \mathrm{A}$ current injection level, a doublet transition at $1.2886 \mathrm{eV}$ is dominant. A second doublet at $1.2893 \mathrm{eV}$ appears at around $0.10 \mu \mathrm{A}$ which shows a superlinear intensity increase with current, whereas the $1.2886 \mathrm{eV}$ transition saturates for currents above $0.1 \mu \mathrm{A}$. Such behavior is typical for exciton and bi-exciton emission of a single QD.

The relationship between these transitions is further analyzed by polarization-dependent measurements shown in Fig. 2, which reveals the paired nature of the XX-X cascade. These findings ultimately identify the low-energy doublet as exciton (X) and the high-energy doublet as anti-binding biexciton (XX) transitions. Correspondingly, the bi-exciton binding energy of $-0.72 \mathrm{meV}$ and fine structure splitting of
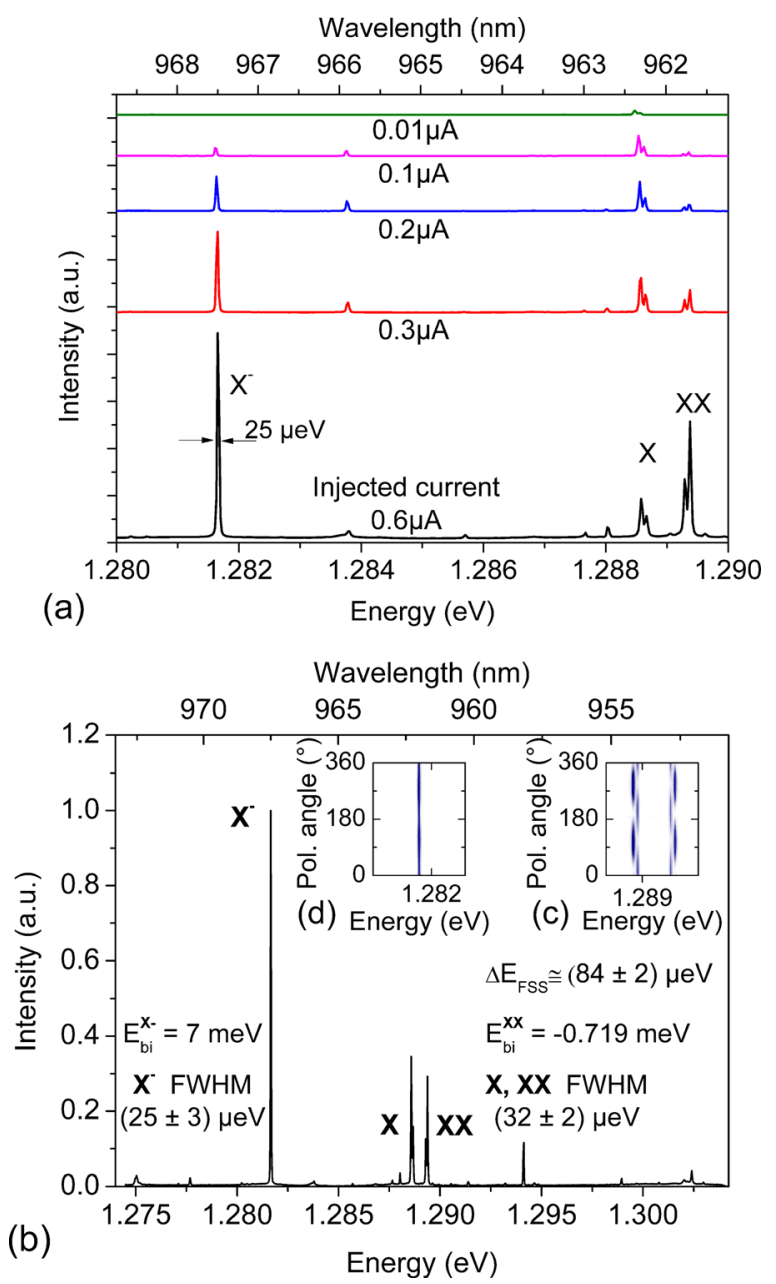

FIG. 2. (a) Injection current dependent $\mu$-EL spectra. Emission lines from exciton $(\mathrm{X})$, bi-exciton $(\mathrm{XX})$, and charged exciton $\left(\mathrm{X}^{-}\right)$transitions are indicated; (b) high resolution spectrum at $0.6 \mu \mathrm{A}$ current injection level with annotations of linewidths and binding energies. Insets (c) and (d) show the polarization dependence.

the exciton levels of $(84 \pm 2) \mu \mathrm{eV}$ are extracted. Both values are in very good agreement with a statistical analysis of QDs for which a correlation of QD size and shape to ground-state optical transition levels was established. ${ }^{26}$ The specific values for the exciton transition energy and bi-exciton binding energy suggest a 3 ML high QD with $10 \mathrm{~nm}$ base length. Spectrally stable transition energies enable to associate the third line at $1.2817 \mathrm{eV}$ to the same QD by comparing the spatial dependence of emission intensity of all lines with regard to objective lens position. Neither FSS nor polarization dependence as seen in Fig. 2(c) is characteristic of this line indicating recombination of charged excitons. Typically, a red shift of the emission wavelength relative to corresponding exciton emission is associated with negatively charged excitons $\left(\mathrm{X}^{-}\right){ }^{26}$ The binding energy of the charged exciton state is $6.96 \mathrm{meV}$ which also agrees well with the estimated size and shape of the QD. A remarkably low resolution limited linewidth of only $25 \mu \mathrm{eV}$ is measured for the $\mathrm{X}^{-}$line confirming that the QD is embedded in a defect-free matrix environment.

Narrow linewidth, spectral separation from other transitions, and high intensity make the $\mathrm{X}^{-}$emission very attractive for generation of single photons on demand. Analysis of 


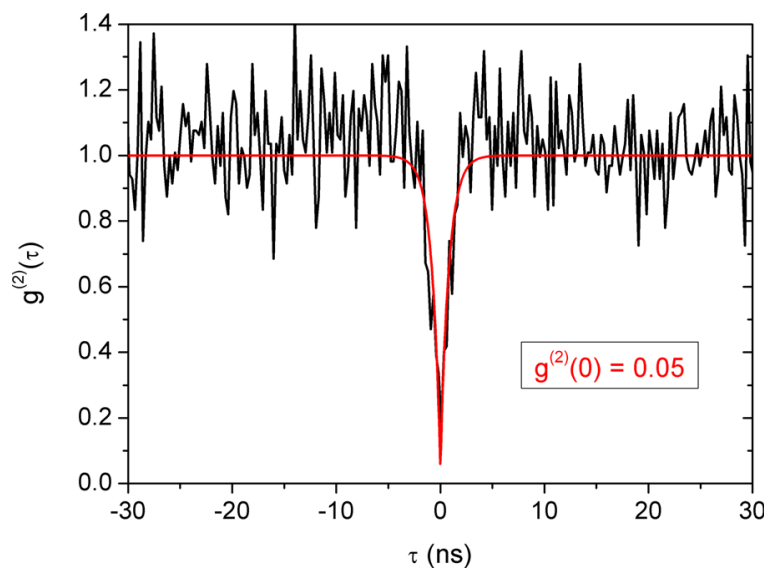

FIG. 3. Trace of the photon auto-correlation measurement signal obtained from the $\mathrm{X}^{-}$emission. The red line is a fit of the second-order auto-correlation function $\mathrm{g}^{(2)}(\tau)$ to raw data.

photon statistics on the $\mathrm{X}^{-}$line is performed with a fiber coupled HBT setup. A fit of the second order autocorrelation function $\mathrm{g}^{(2)}(\tau)$ to the experimental data is shown in Fig. 3 as red line. Clear photon anti-bunching with $\mathrm{g}^{(2)}(0)=0.05$ is obtained at injection current of $0.32 \mu \mathrm{A}$. It is noteworthy that single photon emission with such high purity has not yet been reported for site-controlled QDs. For electrically operated QD-based single photon emitters, the result compares well to best values of $\mathrm{g}^{(2)}(0)=0.02$ measured on devices with randomly distributed QDs. ${ }^{27}$ Moreover, future combining our device concept with suitable microcavity structures will enable the development of deterministic SPS with high outcoupling efficiency.

In conclusion an electrically driven single photon source based on site-controlled QDs is presented. Perfect selfalignment of QDs to current injection path is obtained using a buried stressor technology. Very narrow, resolution-limited emission linewidths down to $25 \mu \mathrm{eV}$ and pure single photon emission yielding $\mathrm{g}^{(2)}(0)=0.05$ are observed. Several excitonic features originating from the same QD are resolved by power- and polarization-dependent $\mu$-electroluminescence spectroscopy.

The authors acknowledge E. Schneiderwind and P. Moser for technical assistance and SFB 787 of DFG for its financial support.

${ }^{1}$ P. Michler, A. Imamoglu, M. D. Mason, P. J. Carson, G. F. Strouse, and S. K. Buratto, Nature (London) 406, 968 (2000).

${ }^{2}$ V. Zwiller, T. Aichele, and O. Benson, New J. Phys. 6, 96 (2004).
${ }^{3}$ D. Bimberg, E. Stock, A. Lochmann, A. Schliwa, J. A. Töfflinger, W. Unrau, M. Münnix, S. Rodt, V. A. Haisler, A. I. Toropov, A. Bakarov, and A. K. Kalagin, IEEE Photon. J. 1, 58 (2009).

${ }^{4}$ A. J. Bennett, D. C. Unitt, A. J. Shields, P. Atkinson, and D. A. Ritchie, Opt. Express 13, 7772 (2005).

${ }^{5}$ A. Lochmann, E. Stock, J. A. Töfflinger, W. Unrau, A. Toropov, A. Bakarov, V. Haisler, and D. Bimberg, Electron. Lett. 45, 566 (2009).

${ }^{6}$ A. J. Shields, Nat. Photonics 1, 215 (2007).

${ }^{7}$ E. Stock, W. Unrau, A. Lochmann, J. A. Töfflinger, M. Özturk, A. I. Toropov, A. K. Bakarov, V. A. Haisler, and D. Bimberg, Semicond. Sci. Technol. 26, 014003 (2011).

${ }^{8}$ T. Heindel, C. Schneider, M. Lermer, S. H. Kwon, T. Braun, S. Reitzenstein, S. Höfling, M. Kamp, and A. Forchel, Appl. Phys. Lett. 96, 011107 (2010).

${ }^{9}$ S. Reitzenstein and A. Forchel, J. Phys. D 43, 033001 (2010).

${ }^{10}$ P. Borri, S. Schneider, W. Langbein, and D. Bimberg, J. Opt. A 8, S33 (2006).

${ }^{11}$ E. Waks, K. Inoue, C. Santori, D. Fattal, J. Vuckovic, G. S. Solomon, and Y. Yamamoto, Nature (London) 420, 762 (2002).

${ }^{12}$ T. Heindel, C. A. Kessler, M. Rau, C. Schneider, M. Fürst, F. Hargart, W. M. Schulz, M. Eichfelder, R. Rossbach, S. Nauerth, M. Lermer, H. Weier, M. Jetter, M. Kamp, S. Reitzenstein, S. Höfling, P. Michler, H. Weinfurter, and A. Forchel, New J. Phys. 14, 083001 (2012).

${ }^{13}$ C. Taylor, E. Marega, E. A. Stach, G. Salamo, L. Hussey, M. Munoz, and A. Malshe, Nanotechnology 19, 015301 (2008).

${ }^{14}$ P. Atkinson, O. G. Schmidt, S. P. Bremner, and D. A. Ritchie, C. R. Phys. 9, 788 (2008).

${ }^{15}$ J. Skiba-Szymanska, A. Jamil, I. Farrer, M. B. Ward, C. A. Nicoll, D. J. P. Ellis, J. P. Griffiths, D. Anderson, G. A. C. Jones, D. A. Ritchie, and A. J. Shields, Nanotechnology 22, 065302 (2011).

${ }^{16}$ M. Felici, P. Gallo, A. Mohan, B. Dwir, A. Rudra, and E. Kapon, Small 5, 938 (2009).

${ }^{17}$ A. Huggenberger, S. Heckelmann, C. Schneider, S. Hofling, S. Reitzenstein, L. Worschech, M. Kamp, and A. Forchel, Appl. Phys. Lett. 98, 131104 (2011).

${ }^{18}$ M. H. Baier, S. Watanabe, E. Pelucchi, and E. Kapon, Appl. Phys. Lett. 84, 1943 (2004).

${ }^{19}$ M. H. Baier, C. Constantin, E. Pelucchi, and E. Kapon, Appl. Phys. Lett. 84, 1967 (2004).

${ }^{20}$ M. Mehta, D. Reuter, A. Melnikov, A. D. Wieck, S. M. de Vasconcellos, T. Baumgarten, A. Zrenner, and C. Meier, Physica E 42, 2749 (2010).

${ }^{21}$ C. Schneider, T. Heindel, A. Huggenberger, T. A. Niederstrasser, S. Reitzenstein, A. Forchel, S. Höfling, and M. Kamp, Appl. Phys. Lett. 100, 091108 (2012).

${ }^{22}$ V. Türck, S. Rodt, O. Stier, R. Heitz, R. Engelhardt, U. W. Pohl, D. Bimberg, and R. Steingrüber, Phys. Rev. B 61, 9944 (2000).

${ }^{23}$ A. Strittmatter, A. Holzbecher, A. Schliwa, J. H. Schulze, D. Quandt, T. D. Germann, A. Dreismann, O. Hitzemann, E. Stock, I. A. Ostapenko, S. Rodt, W. Unrau, U. W. Pohl, A. Hoffmann, D. Bimberg, and V. Haisler, "Site-controlled quantum dot growth on buried oxide stressor layers," Phys. Status Solidi A.

${ }^{24}$ R. L. Sellin, I. Kaiander, D. Ouyang, T. Kettler, U. W. Pohl, D. Bimberg, N. D. Zakharov, and P. Werner, Appl. Phys. Lett. 82, 841 (2003).

${ }^{25}$ A. Strittmatter, T. D. Germann, T. Kettler, K. Posilovic, J. Pohl, U. W. Pohl, and D. Bimberg, J. Cryst. Growth 310, 5066 (2008).

${ }^{26}$ S. Rodt, A. Schliwa, K. Pötschke, F. Guffarth, and D. Bimberg, Phys. Rev. B 71, 155325 (2005).

${ }^{27}$ R. B. Patel, A. J. Bennett, K. Cooper, P. Atkinson, C. A. Nicoll, D. A. Ritchie, and A. J. Shields, Nanotechnology 21, 274011 (2010). 